Participants preferred individual self-monitoring and goal-setting to social features of the app.

There were no significant changes in sedentary time; participants perceived a need for more opportunities for breaks in the workplace. Mental health-related quality of life improved from baseline to month 8 (SF-12 mental component score +1.75 points, 95\% CI $0.28-3.23$ ). Despite interview-reported improvements in health and wellbeing, survey-assessed changes in physical health-related quality of life, perceived stress and perceived productivity were not statistically significant.

Conclusion mHealth technology is a potentially impactful, feasible and acceptable intervention for increasing PA in the police force, particularly for less active officers and staff. The intervention was less useful for reducing sedentary time and the impact on health, wellbeing, stress and productivity is unclear.

\section{OP109 MULTIPLE RISK BEHAVIOUR INTERVENTIONS IN OVERWEIGHT AND OBESE ADULTS: SYSTEMATIC REVIEW AND META-ANALYSIS}

${ }^{1} \mathrm{E}$ South*, ${ }^{1} \mathrm{~N}$ Meader, ${ }^{1} \mathrm{~A}$ Hodkinson, ${ }^{1} \mathrm{H}$ Dale, ${ }^{2} \mathrm{M}$ White, ${ }^{3} \mathrm{C}$ Power, ${ }^{4} \mathrm{M}$ Petticrew, ${ }^{1} \mathrm{~K}$ Wright, ${ }^{1} \mathrm{~A}$ Sowden. ${ }^{1}$ Centre for Reviews and Dissemination, University of York, York, UK; ${ }^{2}$ Centre for Diet and Activity Research, MRC Epidemiology Unit, University of Cambridge, Cambridge, UK; ${ }^{3}$ UCL Great Ormond Street Institute of Child Health, University College London, London, UK; ${ }^{4}$ Faculty of Public Health and Policy, London School of Hygiene and Tropical Medicine, London, UK

\subsection{6/jech-2019-SSMabstracts.69}

Background Risk behaviours (e.g. unhealthy diet, lack of physical activity $(\mathrm{PA})$ ) are associated with increased risk of chronic disease and mortality. The majority of adults in England engage in two or more risk behaviours, which tend to cooccur in individuals. There are also strong socio-economic gradients in key health behaviours. A systematic review of interventions targeting multiple risk behaviours (MRBs) in general adult populations found small improvements in diet, PA and smoking. In this systematic review, we aimed to evaluate the effectiveness of MRB interventions in overweight or obese adults (PROSPERO CRD42016051589).

Methods Six databases were searched up to October 2016 with no language restrictions, and references of relevant systematic reviews were screened. Randomised controlled trials (RCTs) of interventions targeting two or more risk behaviours in obese or overweight adults ( $\geq 16$ years) were eligible if they reported behavioural outcomes. Quality of RCTs was assessed with the Cochrane risk of bias tool. Where possible, random-effects meta-analyses were conducted. Data not included in the meta-analyses were narratively synthesised. Associations between study factors and effectiveness were explored through meta-regression.

Results In total 4392 records were identified, 219 full texts screened and 51 RCTs included. All studies targeted diet and PA, with weight management the most common objective. This contrasts with our review in general adult populations which found smoking and alcohol were often targeted alongside diet and PA and the most common aim was healthy lifestyle promotion. Few interventions included components other than education, training and enablement. There were small changes in fruit and vegetable (MD 27.88 grams, 95\% CI 13.01 to $42.76, \mathrm{I} 2=51.5 \%$ ), fat (SMD $-0.33,95 \%$ CI -0.51 to $-0.15, \mathrm{I} 2=87.7 \%$ ) and calorie intake (MD $-282.89,95 \%$ CI -426.01 to $-139.78, \mathrm{I} 2=91.6$ ) and total PA (SMD 0.21,
$95 \%$ CI 0.01 to $0.41, \mathrm{I} 2=87.2 \%$ ) at the end of interventions. Equivocal benefits were identified for other behavioural outcomes. Generally improvements declined at follow-up and when compared with an active control. We found no associations between study factors and effectiveness at the end of interventions.

Conclusion Interventions were effective for some behavioural outcomes in obese and overweight adults, but improvements were small and declined over time. Studies were limited in their approach, with most adopting 'agentic' strategies which make substantial demands on individuals to initiate and sustain behaviour change. Research is needed into alternative approaches which target the environmental conditions that influence health behaviours and make healthier choices easier for individuals.

\section{OP110 BODY MASS INDEX TRAJECTORIES AND PROSTATE CANCER RISK IN THE EPICAP STUDY}

${ }^{1} \mathrm{C}$ Lavalette*, ${ }^{1} \mathrm{E}$ Cordina Duverger, ${ }^{1} \mathrm{~S}$ Cénée, ${ }^{2} \mathrm{X}$ Rebillard, ${ }^{2,3} \mathrm{PJ}$ Lamy, ${ }^{4} \mathrm{~B}$ Trétarre, ${ }^{1} \mathrm{~F}$ Menegaux. ${ }^{1}$ CESP Team Cancer and Environment, Université Paris-Saclay, Université Paris-Sud, Inserm, Villejuif, France; ${ }^{2}$ Service Urologie, Clinique Beau Soleil, Montpellier, France; ${ }^{3}$ Institut médical d'Analyse Génomique-Imagenome, Labosud, Montpellier, France; ${ }^{4}$ Registre des Tumeurs de I'Hérault, EA 2415, ICM, Montpellier, France

\subsection{6/jech-2019-SSMabstracts.70}

Background High body mass index (BMI) has been inconsistently associated with prostate cancer (PCa) risk. Recent studies suggest that BMI trajectory modeling provides a more robust substitute method to predict cancer risk compared to static measures of BMI. However, only four studies have investigated lifetime $\mathrm{BMI}$ and $\mathrm{PCa}$ risk. We analyzed the effects of life course BMI trajectories on PCa risk based on data from the Epidemiological study of Prostate Cancer (EPICAP).

Methods EPICAP is a French population-based case-control study that enrolled 819 incident cases of PCa diagnosed in 2012 and 2013, aged less than 75 years old and residing in the département of Hérault, France. Controls were 879 agematched men living in the same geographic area. Face to face interviews, using a standardized computerized questionnaire, gathered information about socio-demographic characteristics, medical history, lifestyle factors, physical activity, residential and occupational history. Anthropometric indicators have been collected through the questionnaire (self-report of height at 18 years old and weight every decades) or anthropometric measures at time of interview (height, weight, waist and hip circumferences). BMI trajectories were determined using groupbased trajectory modeling to identify groups of men with similar patterns of BMI change through adulthood. Logistic regression models were used to assess odds ratios (ORs) for the associations between BMI trajectories and PCa risk. Analyses were systematically adjusted for age, family history of PCa and ethnicity. Stratified analyses were conducted by $\mathrm{PCa}$ aggressiveness according to the Gleason score. Seeking for relevant interaction between smoking status and BMI trajectories and given that smoking is a major risk factor for many types of cancer, known to decrease obesity, we performed stratified analyses according to smoking status.

Results We identified four BMI trajectories groups: 'stable normal BMI' (29.7\%), 'normal BMI to overweight' (50.7\%), 'normal BMI to obesity' (17.4\%) and 'overweight to obesity' (2.2\%). Men who had a BMI in the normal range at age 20 
and developed overweight through adulthood had an increased risk of overall (OR 1.27, [0.99-1.62]) and aggressive (OR 1.57, [1.03-2.39]) PCa risk compared to men who maintained a normal BMI. Increased risk of aggressive PCa was also observed among never smokers who developed overweight (OR 3.32, [1.29-8.53]) or obesity (OR 4.19, [1.30-13.51]), but interaction was not significant.

Conclusion Our results suggest that BMI trajectories resulting in overweight or obesity during adulthood are associated with an increased risk of $\mathrm{PCa}$, emphasizing the importance of maintaining a normal BMI throughout adulthood for cancer prevention.

\section{Health Services Research}

\section{OP68 PRIVATE HEALTH INSURANCE STATUS AS A PREDICTOR OF PATIENT EXPERIENCE IN PUBLIC ACUTE HOSPITALS: EVIDENCE FROM A NATIONAL HEALTHCARE SURVEY IN THE REPUBLIC OF IRELAND}

T Huss, C Foley ${ }^{*}$, T Boland, T O'Carroll, D O'Ceallaigh, R Flynn. National Care Experience Programme, Health Information and Quality Authority, Dublin, Ireland

\subsection{6/jech-2019-SSMabstracts.71}

Background In Ireland, patients with private health insurance often receive treatment in publicly-funded hospitals. In the United Kingdom and Australia, a smaller but growing cohort of private patients are also receiving treatment in public hospitals. Surveys have shown that patients with private health insurance (PHI) expect higher standards of care, however, little research has been conducted to examine the association between PHI status and patient experience in public hospitals. This study draws on quantitative and qualitative evidence from the largest healthcare survey in Ireland to examine differences in experience between patients with PHI and those without.

Methods A cross-sectional survey design was used. 27,100 participants in the National Patient Experience Survey who were admitted to a public acute hospital during May 2017 and 2018 responded to a 61-item questionnaire about their journey in hospital. Scales were constructed corresponding to the stages of care: admissions; care on the ward; examinations, diagnosis and treatment; discharge or transfer and overall experience. Multivariate regressions were used to explore differences in patient experience. Qualitative data related to PHI was gathered from three open-ended questions and analysed to explain differences in experience.

Results Controlling for sex, age, length of stay and route of admission, patients with PHI reported more negative experiences across four stages of care and overall experience compared to people without PHI $(\mathrm{p}<0.05) .210$ free-text responses related to PHI. The qualitative analysis highlights that participants' expectations of their PHI benefits were not always fulfilled. Furthermore, a number of patients said that they felt pressured to sign private insurance forms when they were in fact entitled to free public care.

Conclusion In Ireland people with PHI are routinely treated in public hospitals. This study shows that having PHI is a negative predictor of patient experience in public hospitals. Patients expect preferential treatment due to their PHI status and may be disappointed if they do not receive it. The perceived gap between patients' expectations and actual experiences of care influences assessments of satisfaction with a service. Our findings contribute to a greater understanding of the expectations and implications of providing private care in public hospitals, both in Ireland and beyond. The learnings from our study should inform policymakers, service providers and frontline staff in managing patients' expectations in public hospitals.

\section{OP69 IS THERE SOME DEGREE OF UNMET NEED IN PRIMARY CARE?: ANALYSIS OF A PATIENT COHORT ACCESSING A NEW OUT OF HOURS UNITS}

${ }^{1} \mathrm{SJ}$ Kelly*, ${ }^{2} \mathrm{R}$ Ibbotson, ${ }^{3} \mathrm{H}$ Piercy, ${ }^{2} \mathrm{~S}$ Fowler Davis. 'Department of Social Work, Social Care and Community Studies, Sheffield Hallam University, Sheffield, UK; ${ }^{2}$ Department of Allied Health Professions, Sheffield Hallam University, Sheffield, UK; ${ }^{3}$ Department of Nursing and Midwifery, Sheffield Hallam University, Sheffield, UK

\subsection{6/jech-2019-SSMabstracts.72}

Background The increasing demands on Emergency Departments has led to considerable rhetoric on the availability of general practitioner (GP) appointments of which one perceived solution is to offer more out of hours $(\mathrm{OOH})$ care. In England, $\mathrm{OOH}$ healthcare provision is regarded as urgent care only and offered as a mixture of telephone triage, drop-in centres, emergency departments (ED), and triaged appointments. This evaluation describes the patients who used new $\mathrm{OOH}$ appointments offered through the

UK Prime Minister's Challenge Fund scheme which was intended to extend patient access to primary care. The aim of this paper is to report on the demographic profile of attendees and to offer some indication of the impact on ED.

Methods The study used de-identifiable cohort data from 14 months of $\mathrm{OOH}$ appointments offered in 4 units in Sheffield and the responses to the standard NHS patient-opinion questionnaire modified for this programme. Descriptive analysis of the appointment data was conducted. Multivariate logistic regression analysis of the survey data examined the characteristics of the patients who would have gone to the Emergency Department (ED) had the $\mathrm{OOH}$ appointments not been available.

Results There were 24,448 appointments for 19,701 different patients resulting in 29,629 service outcomes (i.e. clinical advice, prescription issued). Six percent of appointments were deemed urgent and two-thirds were non-urgent but needed follow-up. Less than $1 \%$ of appointments were judged inappropriate by the consulting GP. The non-attendance rate was $1.8 \%$. Females accounted for $60 \%$ of all attendances and $70 \%$ in the under 35 age group. The patients from the poorest 5 th of the population used nearly $40 \%$ of the appointments. The patient survey found $\mathrm{OOH}$ appointments were extremely popular - 93\% selecting 'extremely likely' or 'likely' to recommend the service. Regression analysis of patient opinion survey data on whether ED would have been an alternative to the $\mathrm{OOH}$ service found that males, young children, people of Asian heritage and the most deprived were more likely to have gone to ED without this service.

Conclusion Similar to the published literature, the users of the $\mathrm{OOH}$ service were substantially different from in-hours service users; consisting of young adults and children as opposed to the elderly. The findings of this analysis also support the idea that there may be unmet need in the poorest fifth of the population. Future analysis of access to primary care services needs to incorporate patient perceptions and not just statistical data. 